\title{
Development of Logistics for Building Radiation Storm Shelters and their Operational Evaluation
}

Jeffrey A. Cerro

NASA Langley Research Center, Vehicle Analysis Branch

Space 2015, 31 $1^{\text {st }}$ August $-2^{\text {nd }}$ September 2015, Pasadena CA 


\section{O) MIna}

The Storm Shelter Project

- A part of NASA's Advanced Exploration Systems (AES) Radiation Works (RadWorks) Project

- This paper summarizes year 3 of the 3 year RadWorks Storm Shelter program

- FY12 - Trade Space Screening and Concept Selection

- FY13 - Concept development and Use Definition

- FY14 - Complete Concept Development and Usage Evaluation 


\section{OAIAA}

From FY12 Storm Shelter Tradespace Analysis
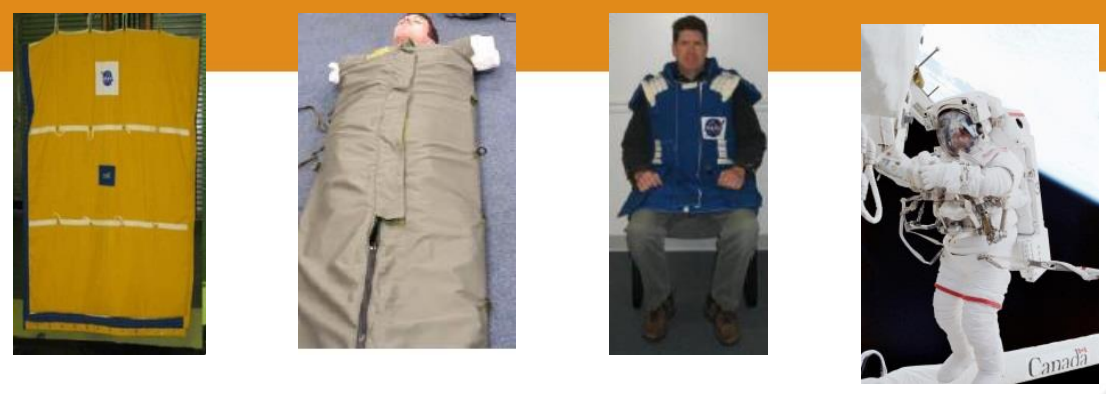

Blankets, Sleeping Bags, Vests, IVA EVA suits,

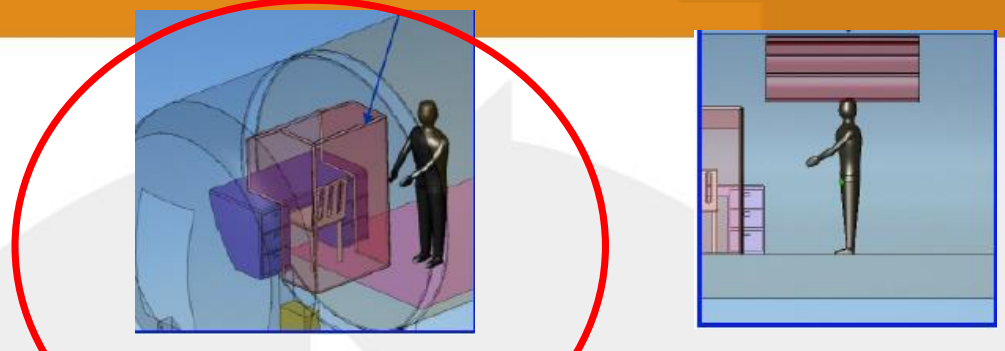

Constructed Sheiter Deployed Shelter

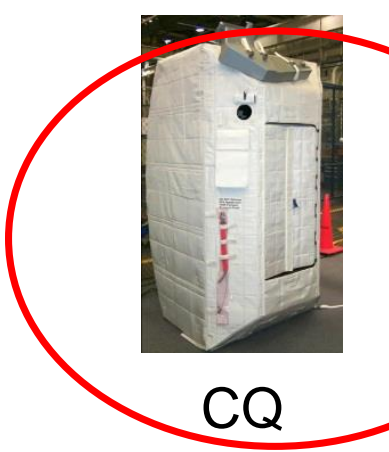

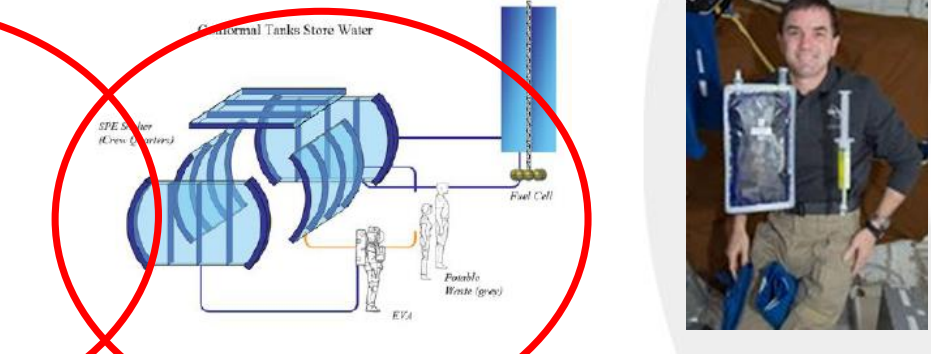

Forward Osmosis

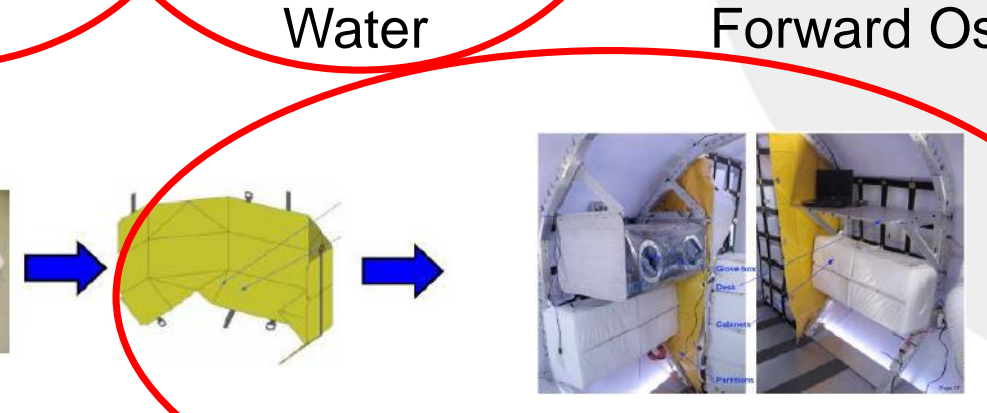

Reconfigured and Repurposed

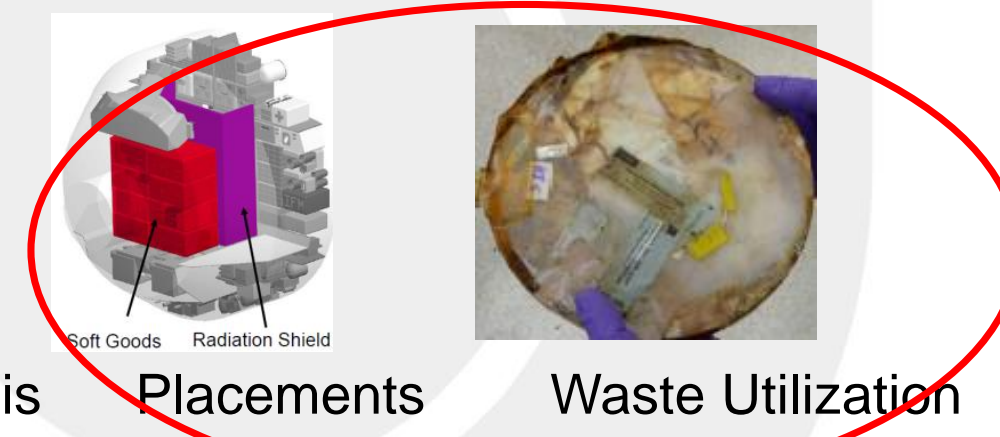

Waste Utilization

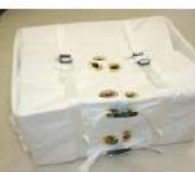




\section{OAIAA}

\section{From FY13 Concept Definition and Development}

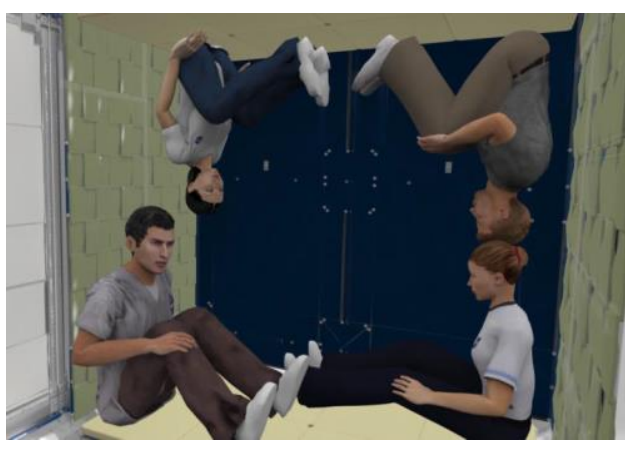

Reused Logistics in a Temporary Centralized Shelter

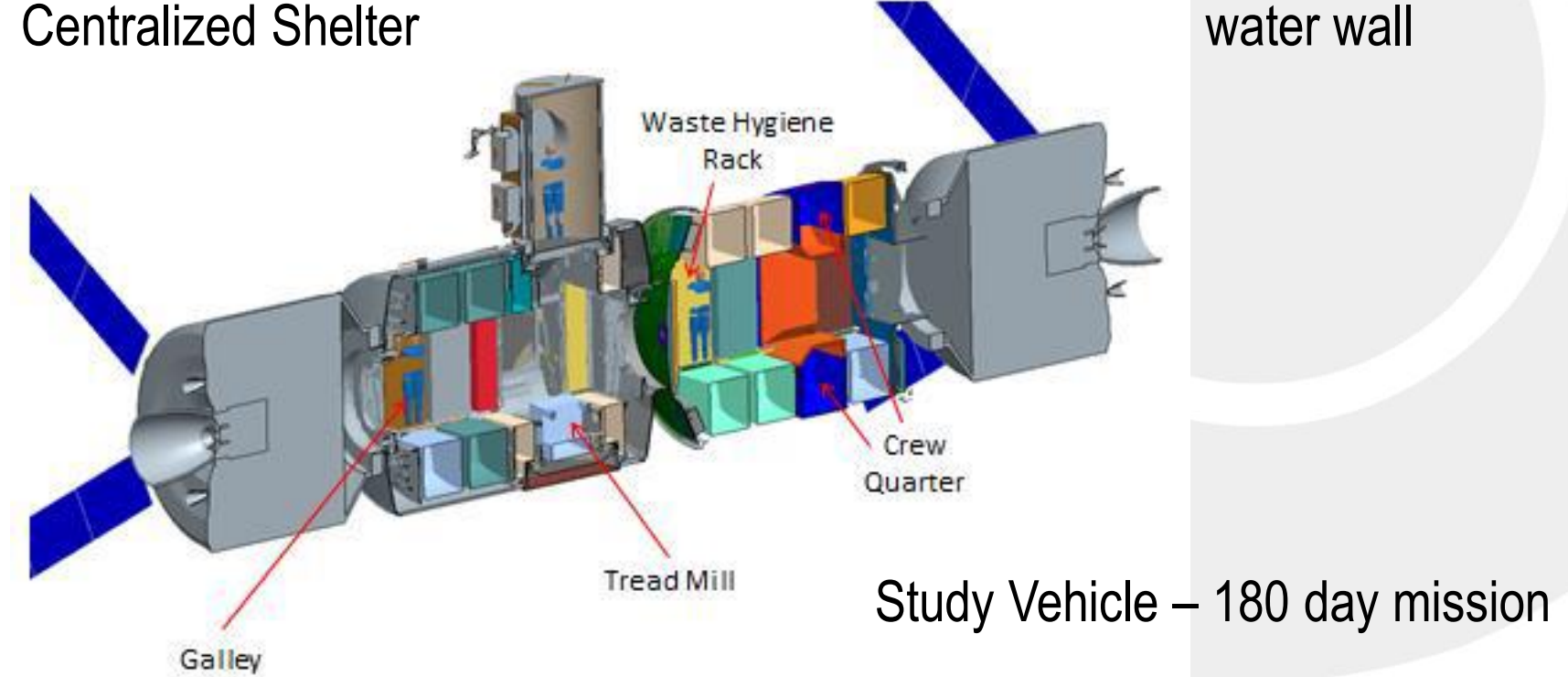

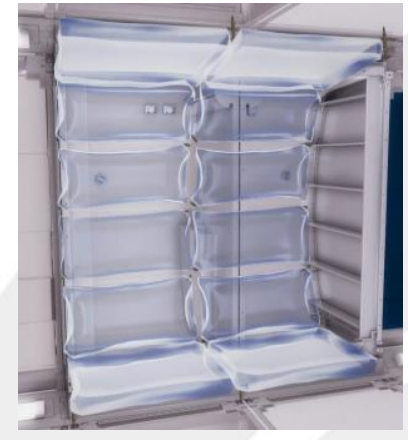

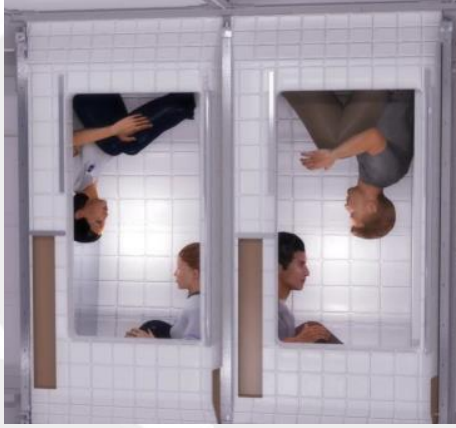

Crew Quarters Protection with a water wall 


\section{OAIAA}

FY14 Concept Development and Testing

Reusable Logistics (RL) and Crew Quarters (CQ) waterwall
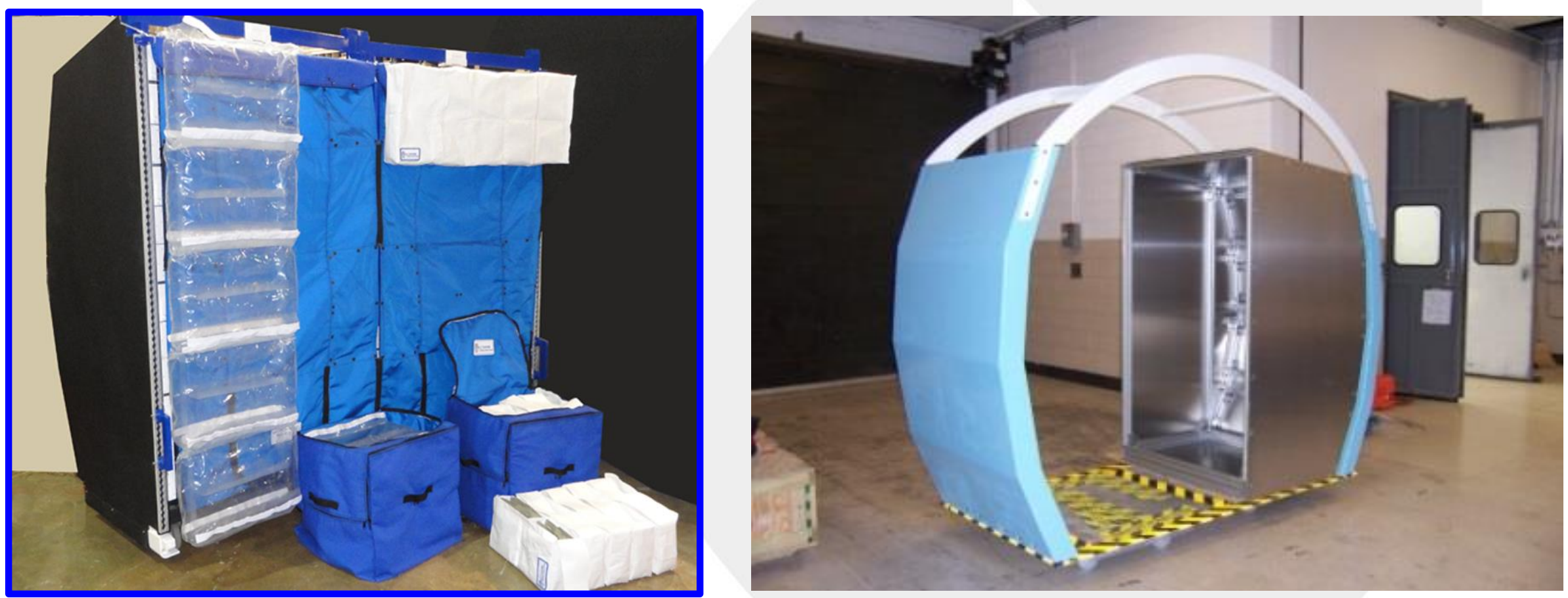
FY14 Concept Development and Testing Reusable Logistics
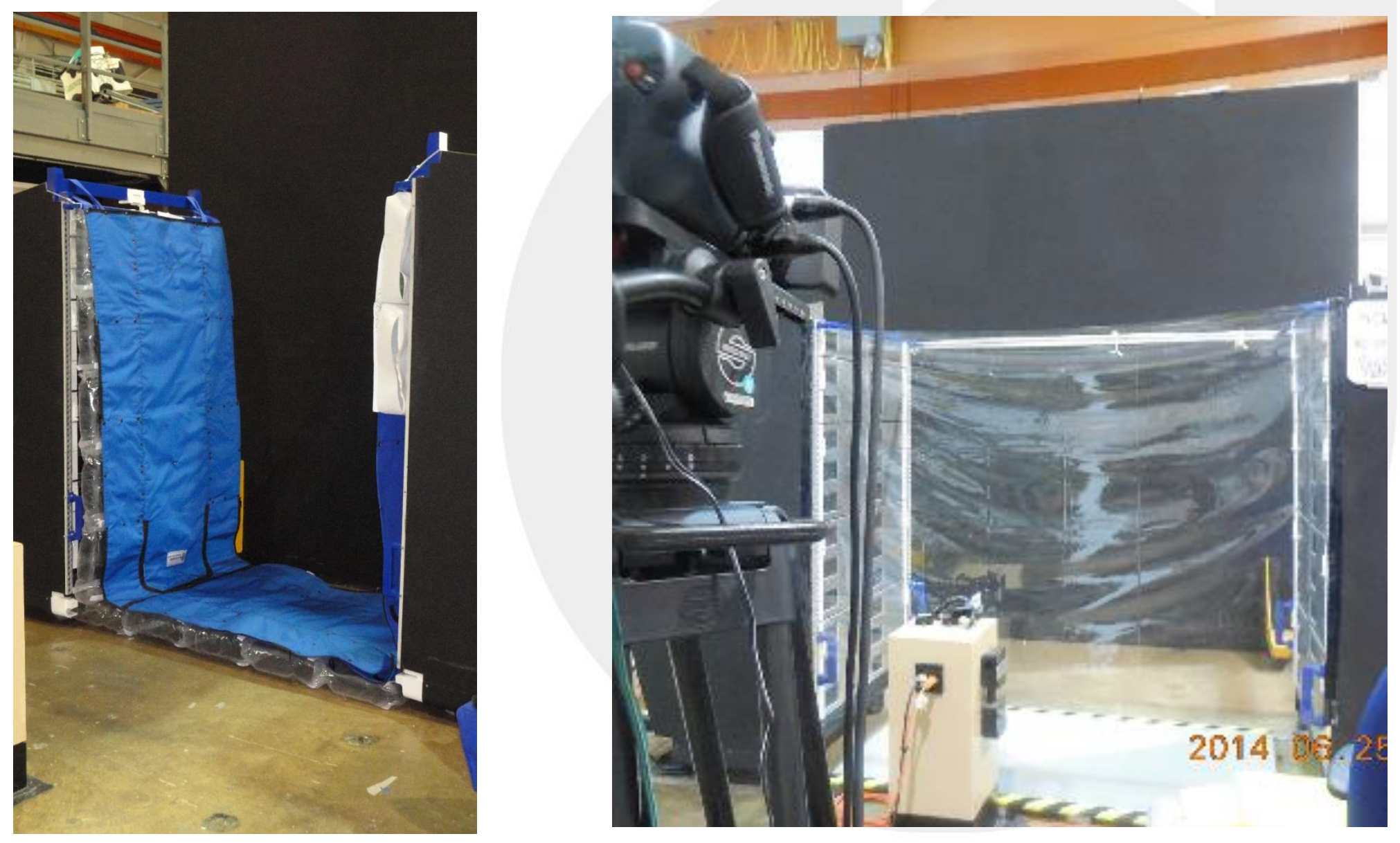


\section{O) MIna}

\section{Logistics for Protection - Food Storage Pouches}

- Storage of food, trash bricks, misc. items

- 3.9 in. $x 8.0$ in wide x 7.8 in. high

- Z-Fold single and double column configurations

- Double Column fills width of MCTB
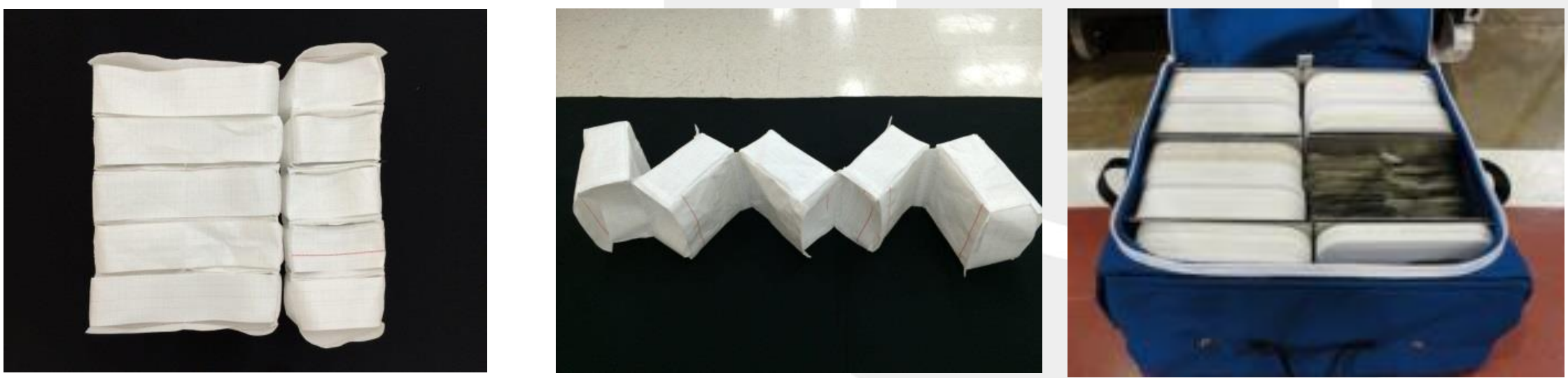


\section{ONIAM}

\section{Logistics for Protection - Contingency Water Containers}

- Approx. 3.9 in. dp x 19.0 in wide x 14.2 in. high

- 4.5 gal containers in single column Z-Fold arrangement

- Air filled mockups used in Human Factors evaluations

- 5 cells fill a double MCTB

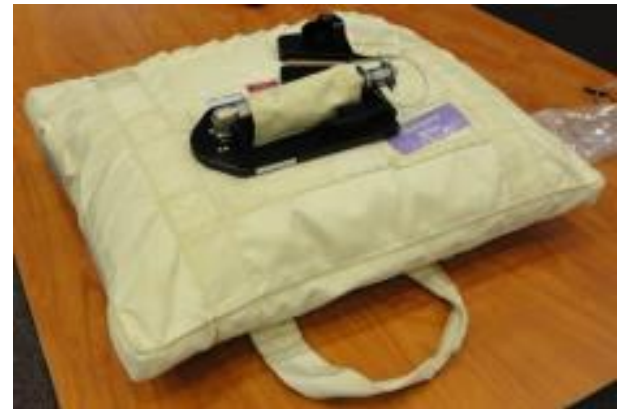

ISS ICWC
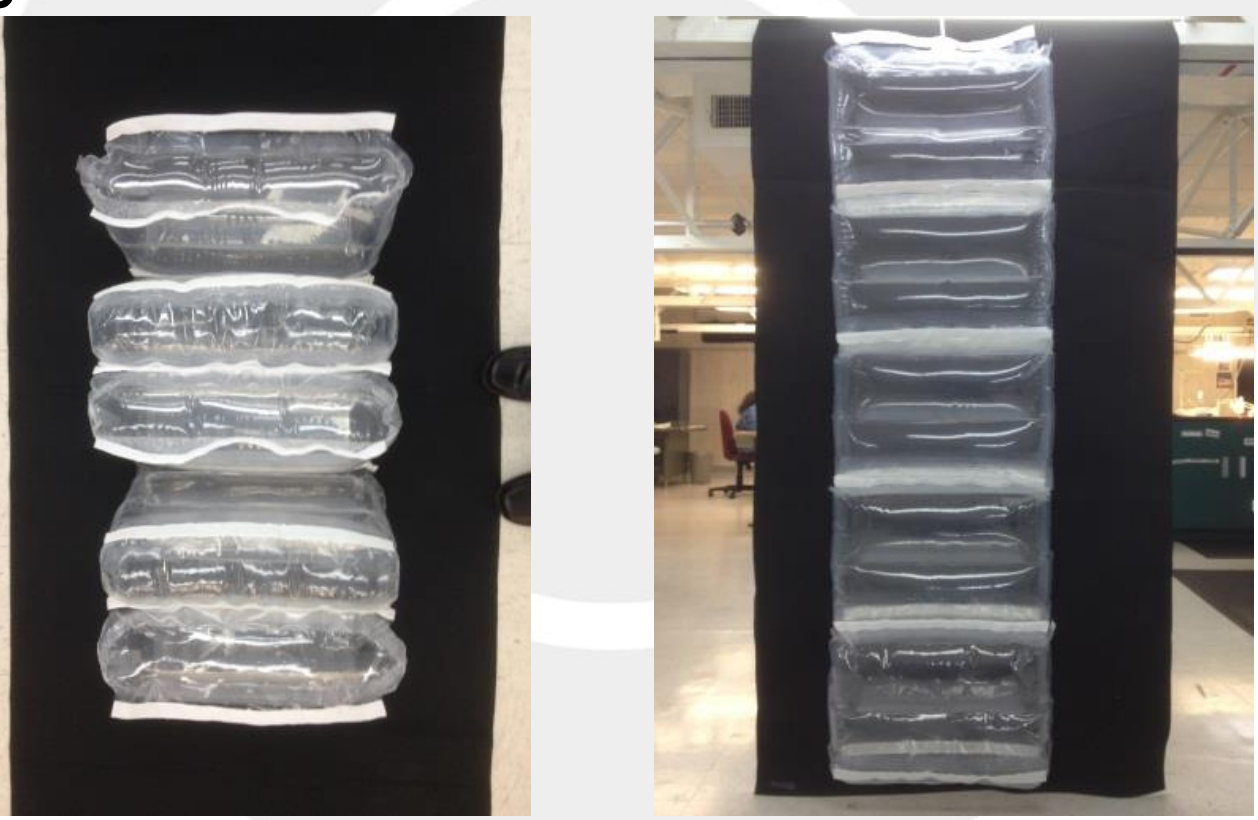

Storm Shelter ICWC 


\section{OAIAM}

\section{Logistics for Protection - Cargo Transfer Bags}

- Utilization of NASA JSC Logistics to Living Program Modified Cargo Transfer Bags (MCTB's)

- Provides

- Initial stowage of logistics

- backing / covering face for logistics placement

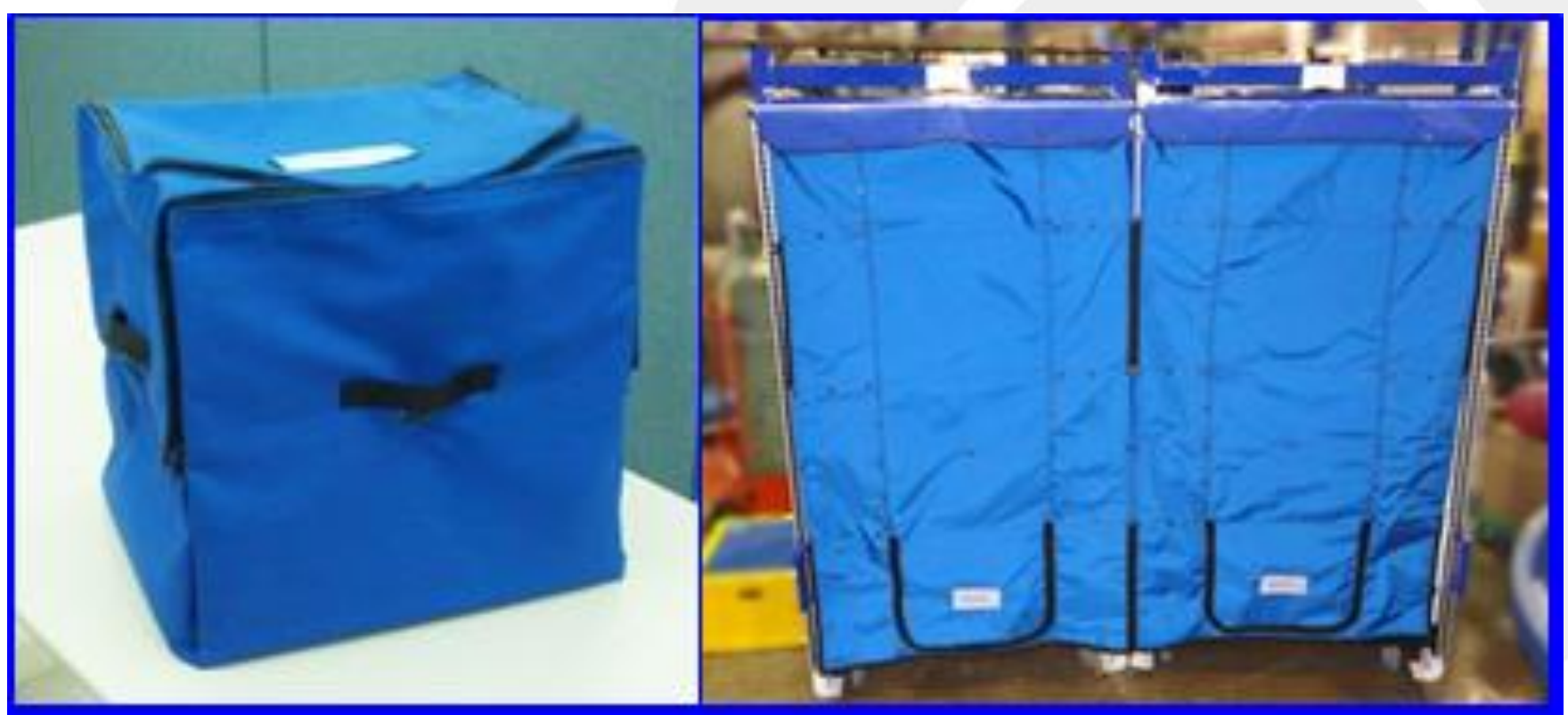




\section{OAIAA}

\section{Logistics for Protection - Common Protection Containers}

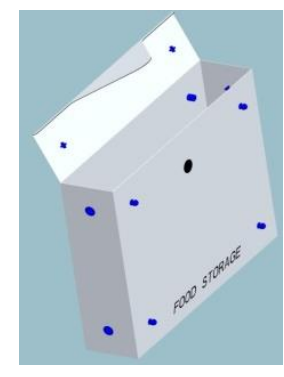

Dry Item Storage
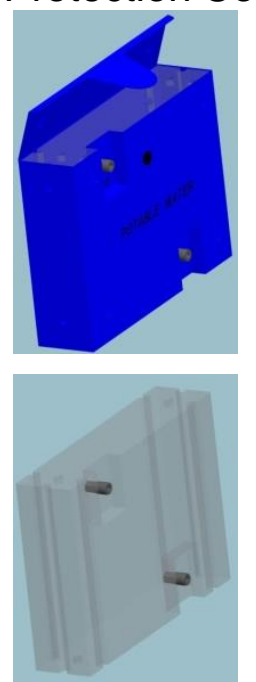

Potable Water - PED
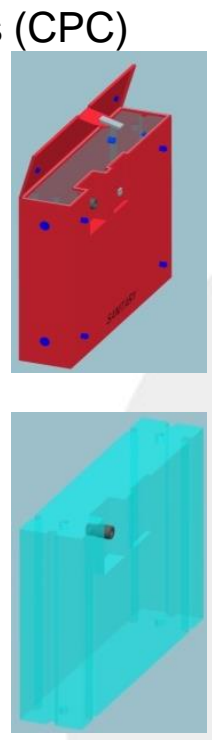

Wet Trash Storage

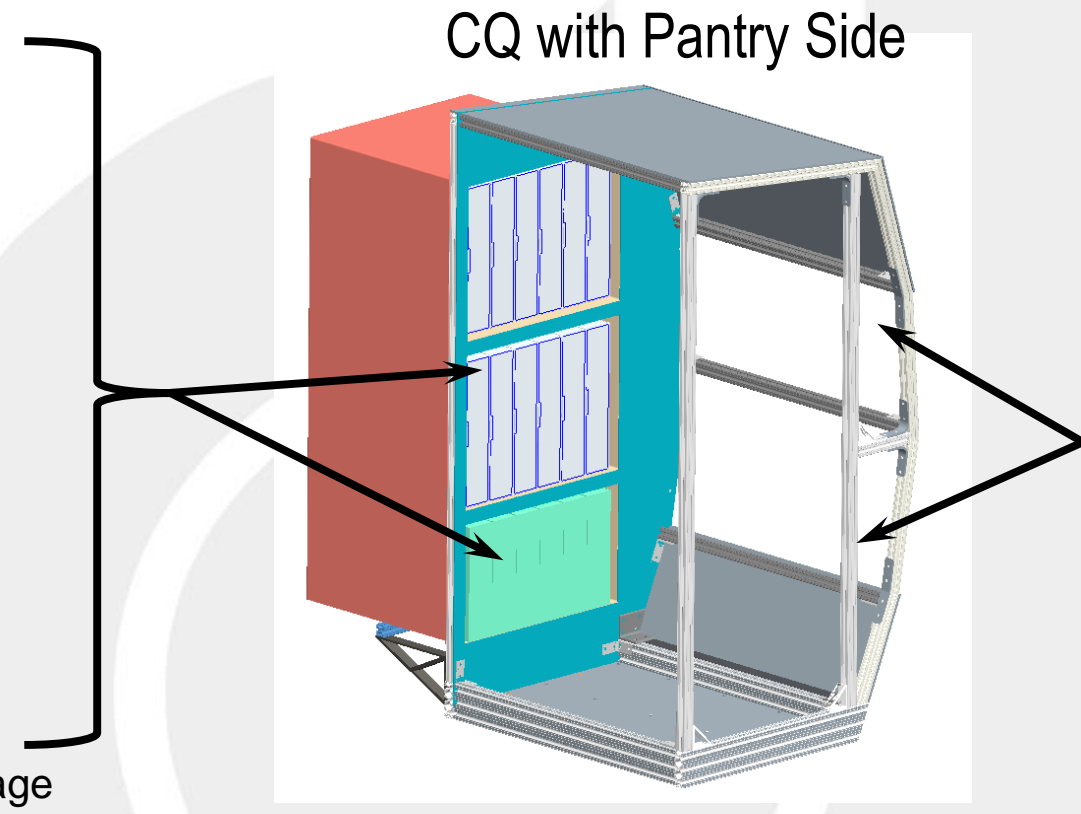

Waterwall

Feedthru

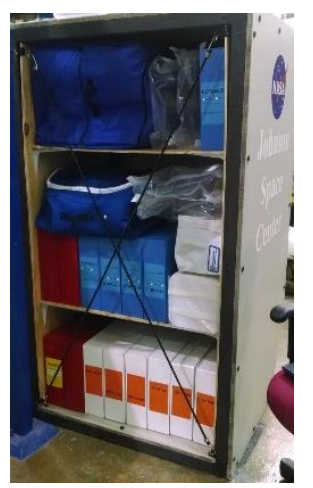

ISPR Staging area, CPC's MCTB's, misc. logistics 


\section{Logistics for Protection - Miscellaneous items}

Thermostabilized food

Storm Shelter "Bulk Overwrap Bag"

Thermostabilized food and Heat Melt Compacted Bricks in FSP's

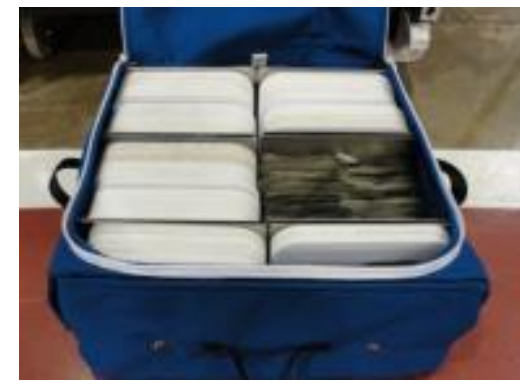

Staging of FSP packaged items in an MCTB 


\section{ONIAM}

\section{FY14 Concept Development and Testing Crew Quarters (CQ) Based}

- Waterwall and Pantry features

- Bladder and Positive Expulsion Device Demonstration

- Automated Water Management and Potable Water Dispenser mockup interface

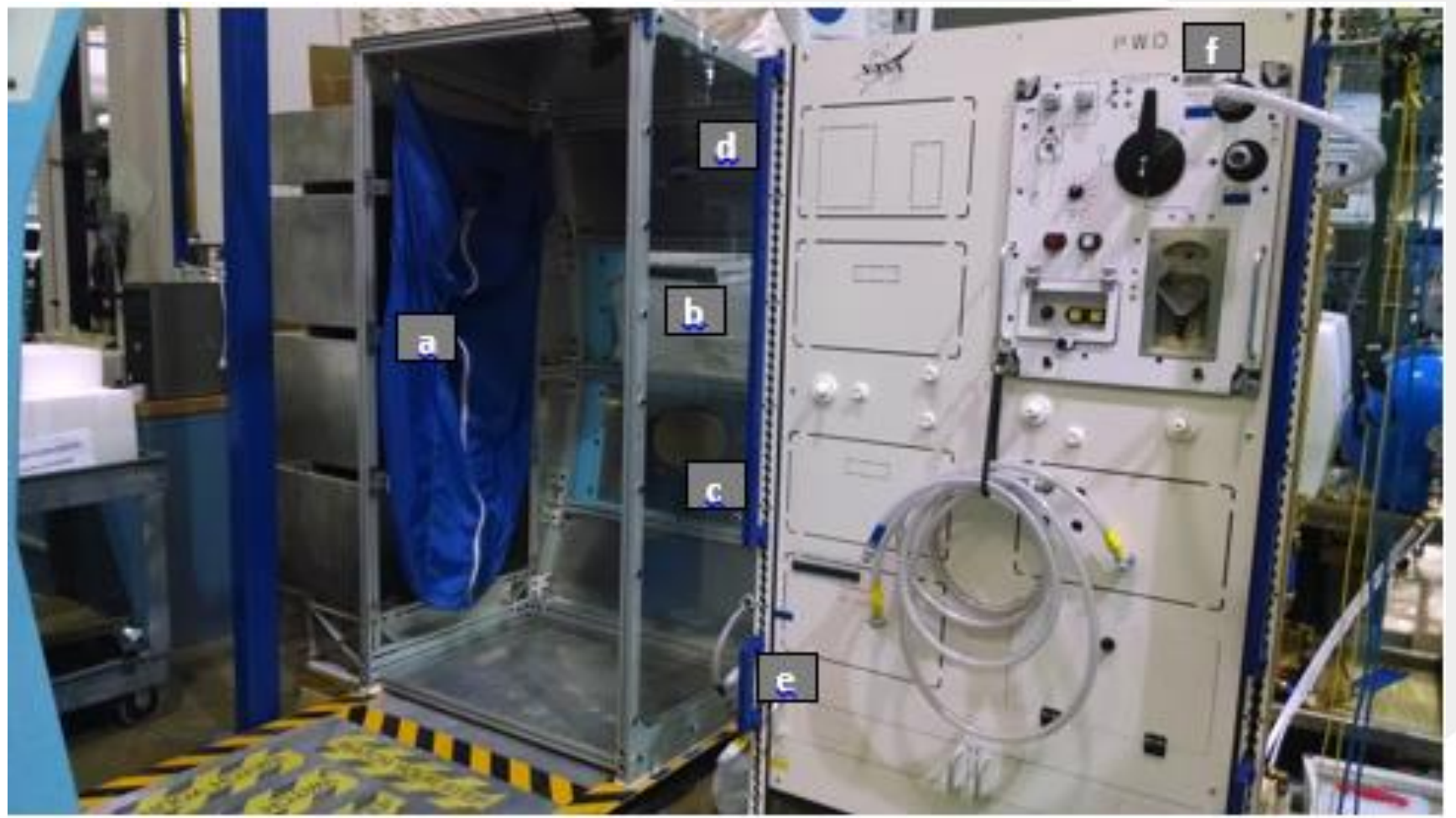




\section{Waterwall Component Types}

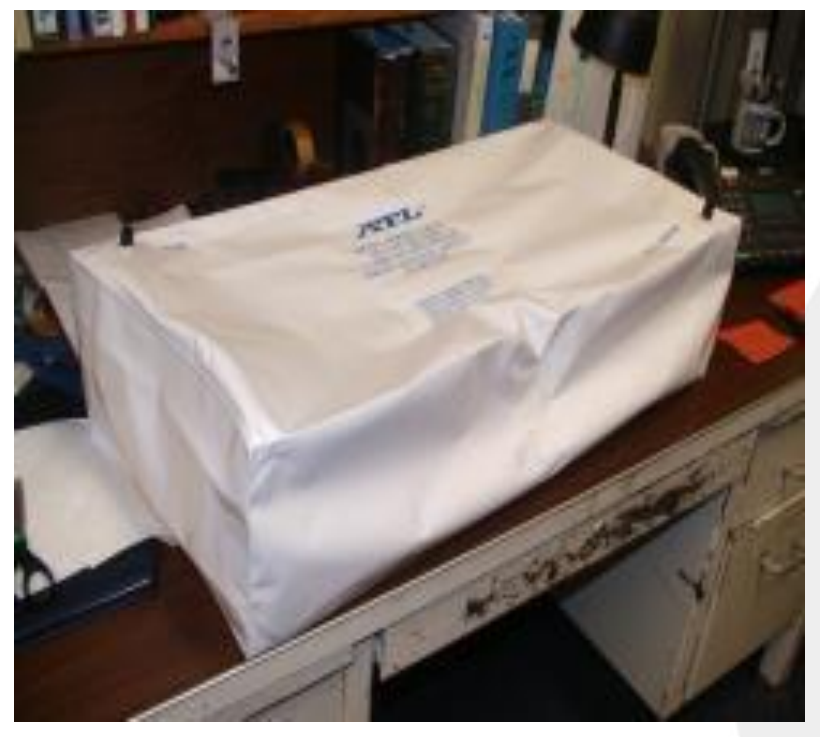

Bladder

- 8.0 in. dp. $\times 14.0$ in. high $\times 30.0$ in. wide

- 14.5 gal. capacity

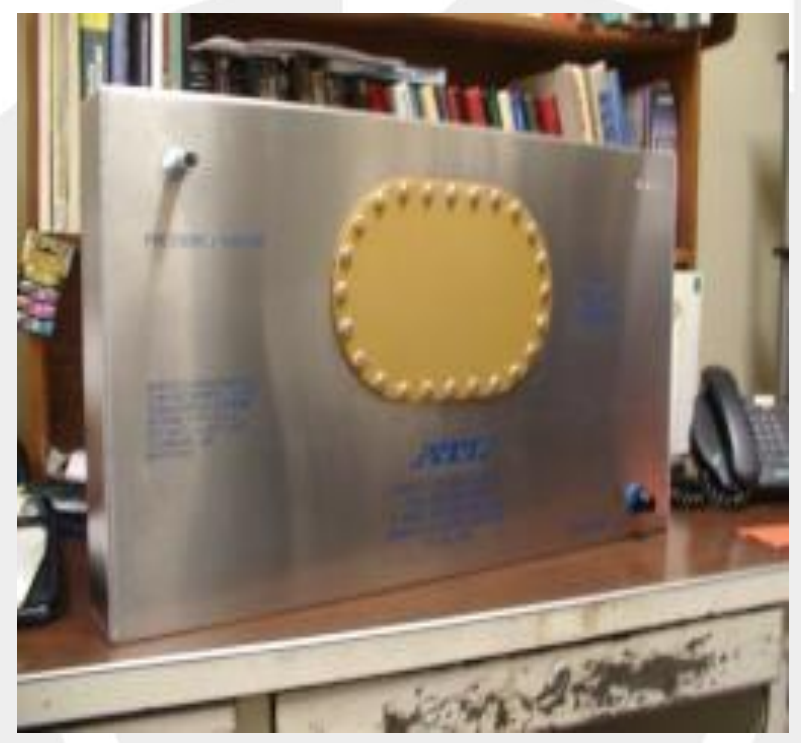

Positive Expulsion Device

- 3.5 in. dp. x 16.3 in. high $\times 29.8$ in. wide

- 6.8 gal. capacity 


\section{Water Wall - Tablet Interface}

NASA Langley Research Center AES RadWorks Water Wall

Water Management System

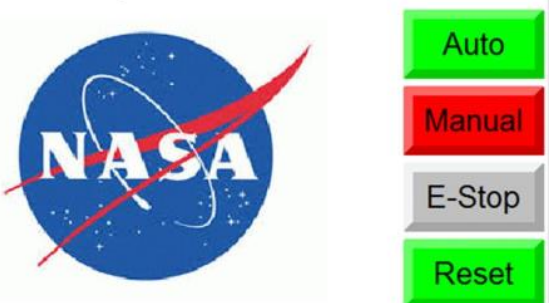

Main
Seq

Manual

Status

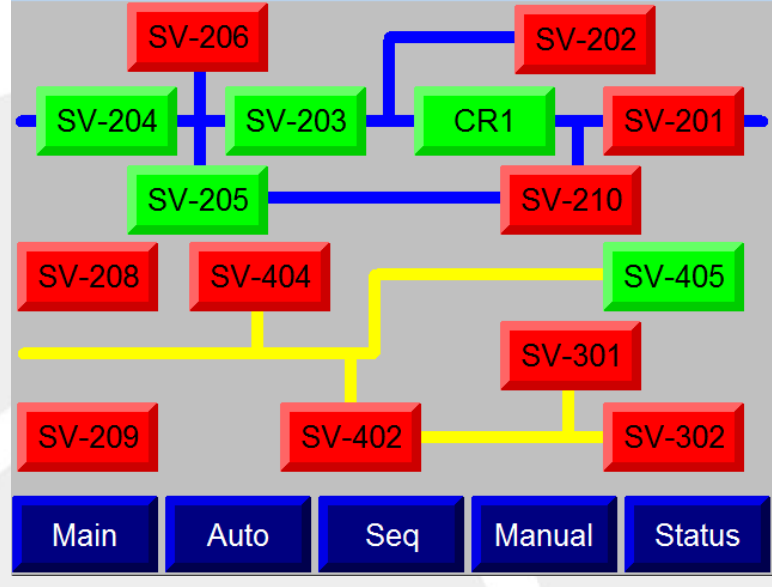

Manual

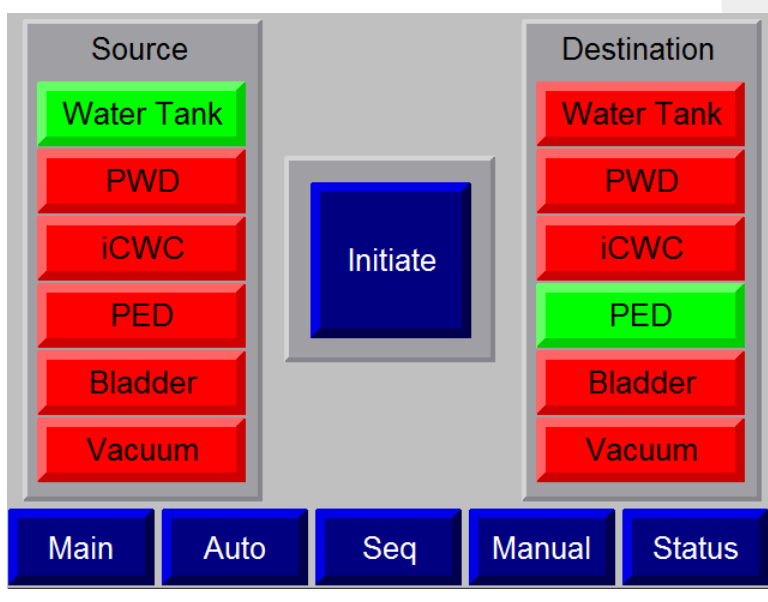

Automatic

\begin{tabular}{|c|c|c|c|c|c|}
\hline \multicolumn{2}{|c|}{ MV-207 } & \multicolumn{2}{|c|}{ MV-403 } & \multicolumn{2}{|c|}{ PT-202 } \\
\hline SP & NN.NN & $\mathrm{SP}$ & NN.NN & \multicolumn{2}{|c|}{$\frac{\text { NN.NN PSIA }}{\text { PT-401 }}$} \\
\hline PV & NN.NN & PV & NN.NN & \multicolumn{2}{|c|}{ NN.NN PSIA } \\
\hline CV & NN.NN & CV & NN.NN & \\
\hline & Auto & & Auto & NN.N & GAL \\
\hline & Remote & & Remote & $\begin{array}{c}\text { Water } \\
\text { Detected }\end{array}$ & \\
\hline $\mathrm{Me}$ & & & Seq & Manual & Status \\
\hline
\end{tabular}

Status 


\section{Additional CQ operations - Pantry}

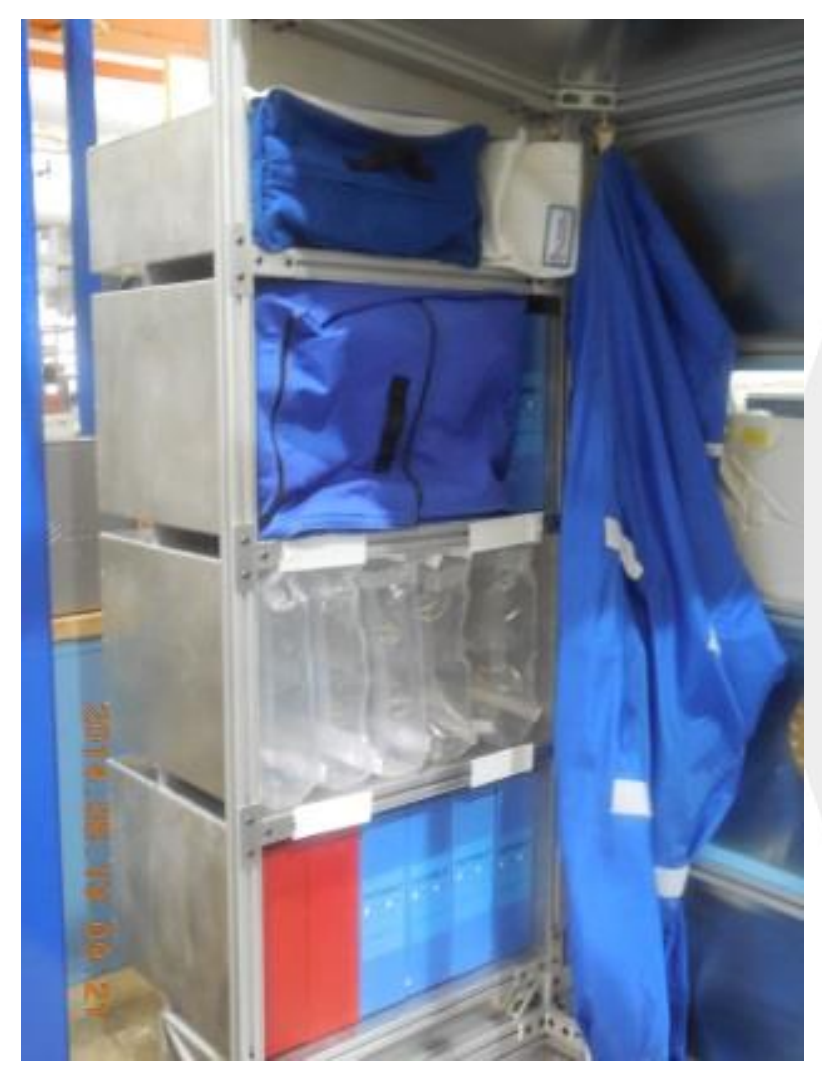

- Use of common dimension and miscellaneous fill logistics in a CQ Pantry 


\section{Human Factors Evaluation - Process}

- NASA LaRC Institutional Review Board approved evaluation process

- 12 teams

- 6 Two crew teams - RL testing

- 6 Single crew - RL testing

- 8 Single crew - CQ testing

- For each experiment

- $1 / 2$ provided general guidance and written instructions

- $1 / 2$ provided general guidance only 


\section{Human Factors Evaluation - Test Sessions}

- 10 and 20 min time proposed as the SPE warning period (desired time to complete shelter build)

- Instructed to behave as if in $0 \mathrm{~g}$ environment.

- Consideration given also to shelter quality to balance the time criterion

- Minimize gaps and poor distribution/placement of protection items

- 3 sessions per experiment run, to assess learning improvement effect 


\section{Human Factors Evaluation - Measures}

- Data captured

- Video data

- Time on task

- Reference to instruction

- Motion data - Actigraphy results 


\section{Human Factors Evaluation - subject evaluations}

- Post test questionnaires to quantify crew assessment of shelter builds

- Temporal demand, acceptability of completion in 10 / 20 mins

- Mental demand

- Physical demand

- Perceived performance

- Effort

- Frustration

- Acceptability

- Exertion/Discomfort

- Dexterity Required

- Envisioned vs evolved assessment

- Degree of protection (completeness)

- Appropriateness of instructions

- CQ software useability 


\section{Human Factors Evaluation - Measurements}

Example factor measurements - Reconfigurable Logistics

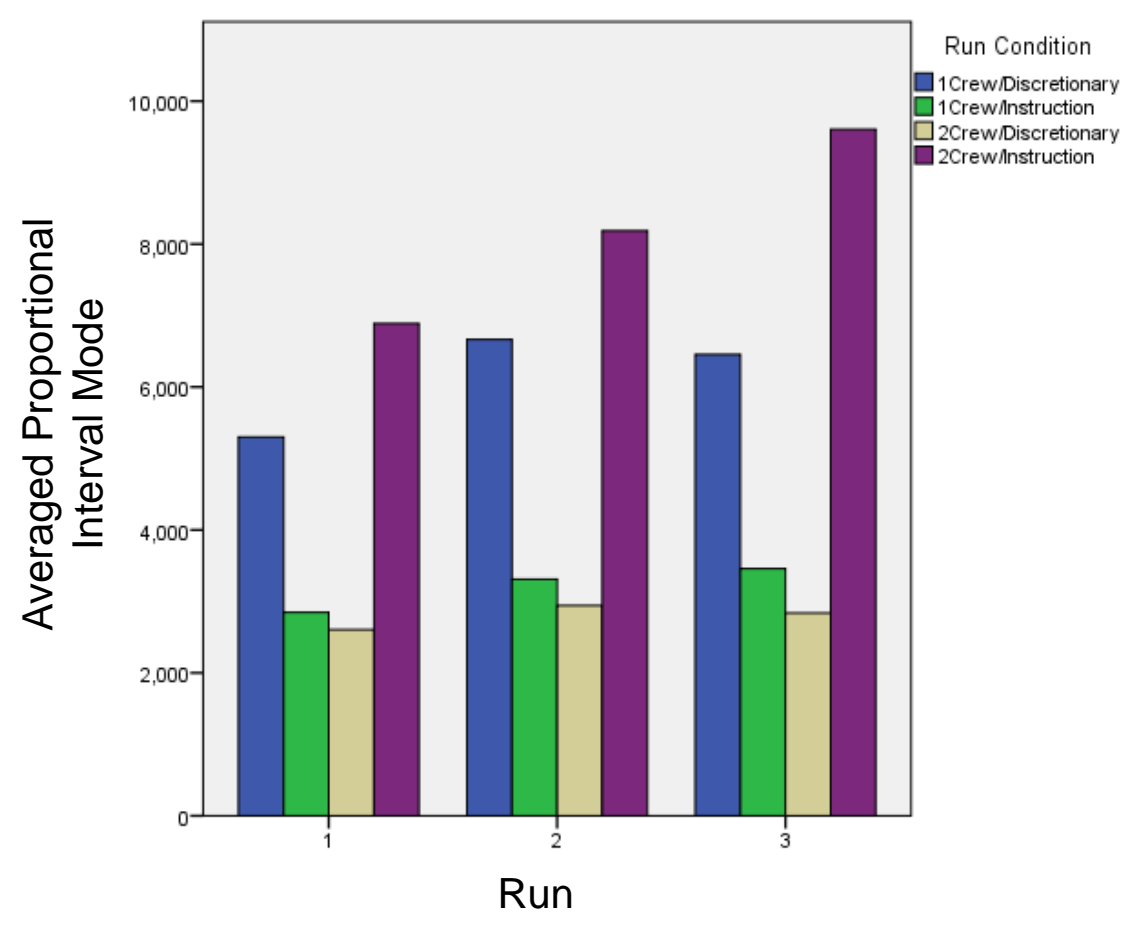

Actigraphy results

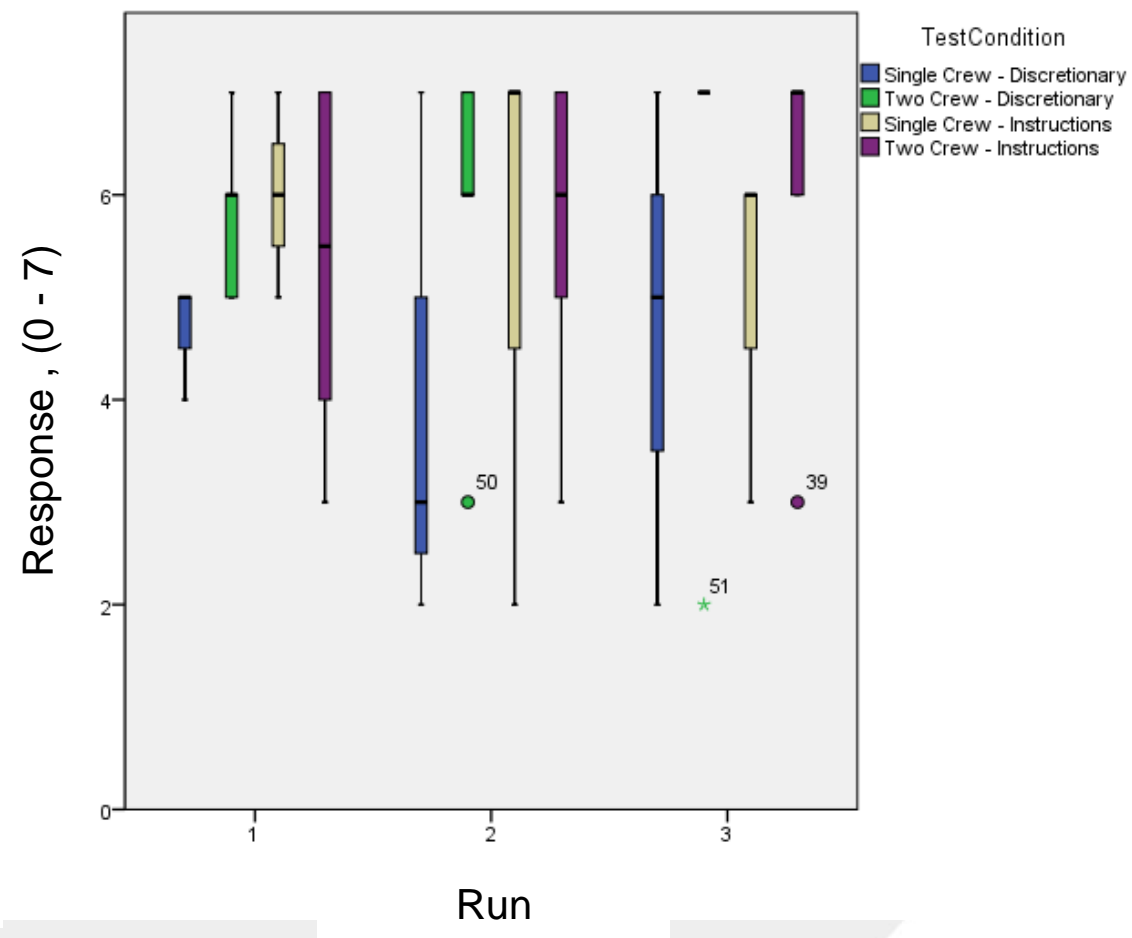

Acceptability to complete in 20 minutes 


\section{Human Factors Evaluation - Measurements}

\section{Example factor measurements - Crew Quarters}

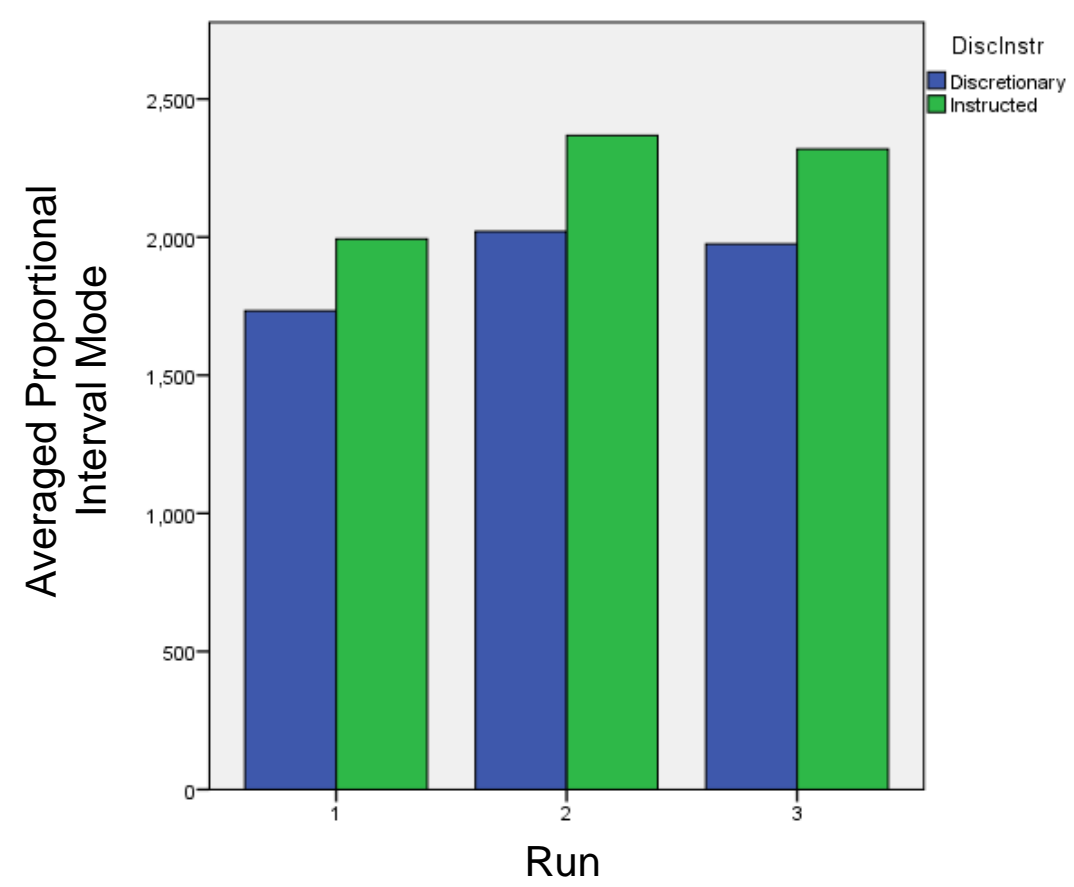

Actigraphy results

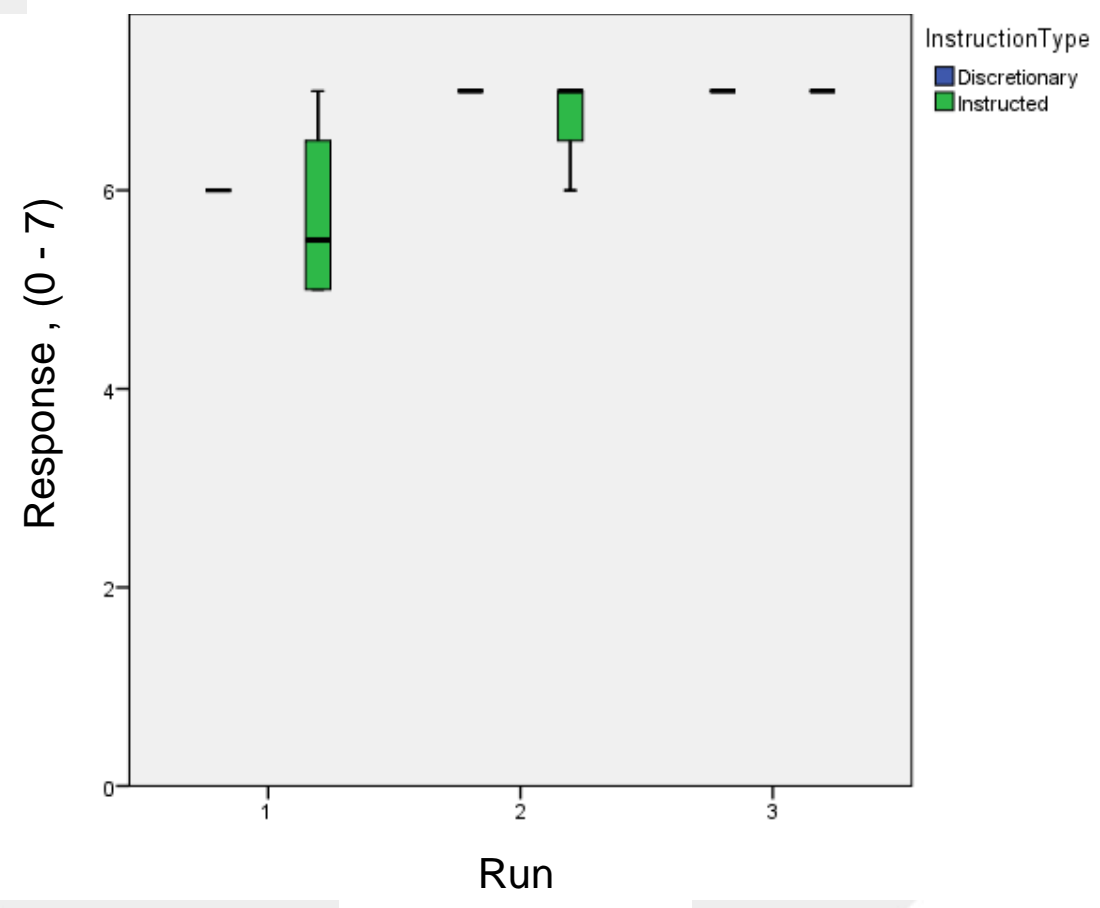

Acceptability to complete in 20 minutes 


\section{Human Factors Evaluation - Observations}

- 20 min build time - acceptable

- 10 min build time - acceptable for 2-Crew Reconfigurable Logistics shelter build, not acceptable for Crew Quarters water wall shelter

- 2-Crew Reconfigurable Logistics shelter build

- Less physical and mental exertion, more confidence in finished quality

- Certain tasks proceed as well with / without instruction

- Pantry fill

- Instructions

- Useful for complex operations

- can slow operations in intuitive procedures

- Water wall operations

- Flagged with some degree of ambiguity in the tablet interface

- Repetitive task training definitely shown to improve speed/quality

- Design for Operations - proven as a good practice ( Crew involvement in design 22 features ) 


\section{Additional and Future Activities}

Demonstration area integration
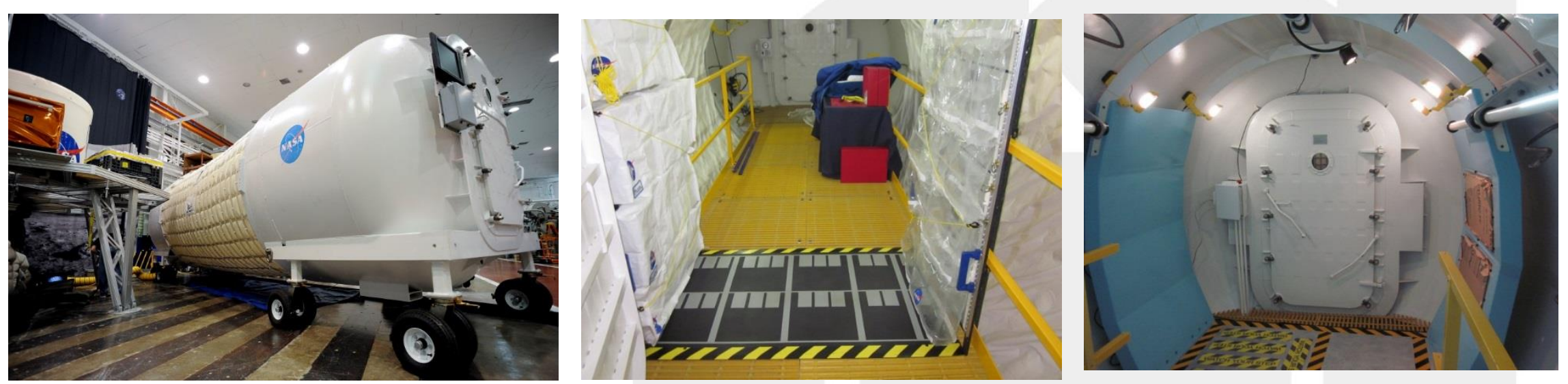

- Completed Integration into LaRC $3 \mathrm{~m}$ dia. X 10m lg. Inflatable Habitat 


\section{Additional and Future Activities}

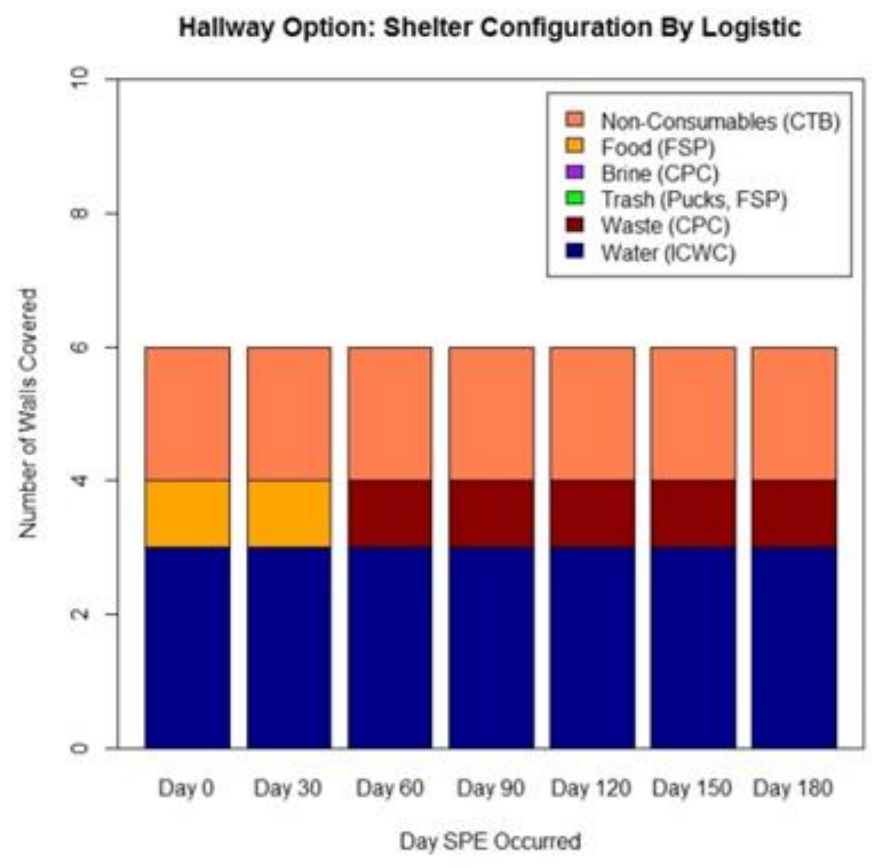

Discrete Event Simulation of Mission Operations
Upper 95th Percentile REID vs. Effective Dose Long missions (365 and 600 days) - Solar maximum GCR environment with SPE (August '72 King fit) beyond low Earth orbit (LEO)

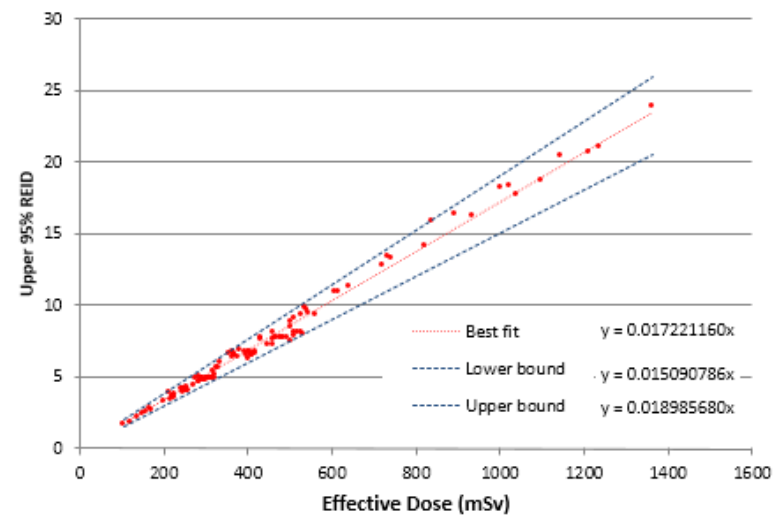

Application of REID to Concept Development 


\section{Conclusions}

- Logistics materials and operating equipment fabricated and tested

- RL (logistics) and CQ (waterwall / logistics)

- Finished components integrated into a demonstration habitat facility available for future taskwork.

- Component shape/size requirements of common dimension are useful, likely a function of chosen habitat design/layout

- Design shelter building for intuitive operations

- Design with increased user involvement as habitat concepts become clearer

- Provide adequate training

- Greater development of validated DES models by operations testing would be a useful habitat design resource

- Influence of Zero gravity desired

- ISS, neutral buoyancy, parabolic flight 


\section{Acknowledgement - The Storm Shelter Team}

- Project Manager - Bobbie Gail Swan NASA / JSC

- Principal Investigator - Eddie Semones, NASA / JSC

\begin{tabular}{|c|c|c|c|}
\hline Last & First & Responsibility & \begin{tabular}{|c|} 
Supporting Organization \\
\end{tabular} \\
\hline Abston & Lee & $\begin{array}{l}\text { HDU CAD model } \\
\text { developer }\end{array}$ & NASA LaRC - Engineering Directorate \\
\hline Albertson & Cindy & Analyst - CQ Lead & NASA LaRC - Systems Analysis and Concepts Directorate \\
\hline Andrews & Rob & Fabrication & NASA LaRC - Engineering Directorate \\
\hline Araiza & Sherry & Resource Analyst & NASA LaRC - Office of the Chief Financial Officer \\
\hline Castle & David & Design & NASA LaRC - Engineering Directorate \\
\hline Cerro & Jeff & Technical Lead & NASA LaRC - Systems Analysis and Concepts Directorate \\
\hline Clark & Terry & $\begin{array}{l}\text { Design - Crew } \\
\text { Quarters }\end{array}$ & NASA LaRC - Engineering Directorate \\
\hline Clowdsley & Martha & Radiation Analysis & NASA LaRC - Research Directorate \\
\hline Connolly & Heidi & $\begin{array}{l}\text { Configuration } \\
\text { Management }\end{array}$ & NASA LaRC Safety and Mission Assurance Office \\
\hline Gallegos & Adam & CAD modeling & Lockheed Martin Corp. \\
\hline $\begin{array}{l}\text { Hintermeist } \\
\text { er }\end{array}$ & Nicole & Scheduling & NASA LaRC Space Technology and Exploration Directorate \\
\hline \begin{tabular}{|l|l} 
James & \\
\end{tabular} & Sam & Fabrication & NASA LaRC - Engineering Directorate \\
\hline Jordan & Tommy & Technical Lead & NASA LaRC - Engineering Directorate \\
\hline Kevin & Krohto & Fabrication & NASA LaRC - Engineering Directorate \\
\hline Langford & Mike & Design & NASA LaRC - Engineering Directorate \\
\hline Latorella & Kara & Human Factors & NASA LaRC - Research Directorate \\
\hline Le Boffe & Vincent & Design/Fabrication & NASA LaRC - Engineering Directorate \\
\hline McLain & Kevin & Fabrication & NASA LaRC - Engineering Directorate \\
\hline
\end{tabular}




\section{Acknowledgement - The Storm Shelter Team}

\begin{tabular}{|c|c|c|c|}
\hline Last & First & Responsibility & Supporting Organization \\
\hline Moore & David & $\begin{array}{l}\text { Deputy Project Manager - } \\
\text { RadWorks }\end{array}$ & NASA LaRC - Engineering Directorate \\
\hline Moses & Bob & SE\&I & NASA LaRC - Engineering Directorate \\
\hline Neubauer & Ken & Risk Management & NASA LaRC Safety and Mission Assurance Office \\
\hline Noble & Lee & Systems Engineering and Int & NASA LaRC - Engineering Directorate \\
\hline Oneil & Teresa & Lab Activities & NASA LaRC - Research Directorate \\
\hline Piske & Andrew & Configuration Management & NASA LaRC Safety and Mission Assurance Office \\
\hline Scharf & Shawn & Risk Management & NASA LaRC Safety and Mission Assurance Office \\
\hline Schneider & Nigel & Design & NASA LaRC Engineering Directorate \\
\hline Shea & Ed & Risk Management & NASA LaRC Safety and Mission Assurance Office \\
\hline Simon & Matt & $\begin{array}{l}\text { Systems Engineering, Habitat } \\
\text { Design }\end{array}$ & NASA LaRC - Systems Analysis and Concepts Directorate \\
\hline Singleterry & Robert & Radiation Analysis & NASA LaRC - Research Directorate \\
\hline Smith & Don & Fabrication & NASA LaRC - Engineering Directorate \\
\hline Thibeault & Sheila & Radiation/Design Consulting & NASA LaRC - Research Directorate \\
\hline Tomek & Debi & LaRC Project Manager & NASA LaRC - Space Technology and Exploration Directorate \\
\hline Vitullo & Nick & Design/Radiation Analysis & NASA LaRC Engineering Directorate \\
\hline Walker & Steve & Radiation Analysis & NASA LaRC - Research Directorate \\
\hline Watson & Judith & $\begin{array}{l}\text { Reconfigurable Logistics } \\
\text { Concept Design }\end{array}$ & NASA LaRC - Research Directorate \\
\hline Werner & Jeff & Mechanical/Plumbing & NASA LaRC - Engineering Directorate \\
\hline Whitlow & Dustin & Design/Radiation Analysis & TEAMS2 - NASA LaRC - Engineering Directorate \\
\hline Wittkopp & Charles & Design/Radiation Analysis & NASA LaRC - Engineering Directorate \\
\hline Mclain & Kriss & Fabrication & NASA LaRC - Engineering Directorate \\
\hline Hester & Jack & Fabrication & NASA LaRC - Engineering Directorate \\
\hline Guild & Kathryn & Media Services & NASA LaRC - Office of the Chief Information Officer \\
\hline Lorkiewicz & Rob & Media Services & NASA LaRC - Office of the Chief Information Officer \\
\hline Tury & Jacob & Fabrication & NASA LaRC - Engineering Directorate \\
\hline Mayhew & Frank & Fabrication & NASA LaRC - Engineering Directorate \\
\hline
\end{tabular}




\section{O)}

The World's Forum for Aerospace Leadership 